Strahlenther Onkol 2012 · 188:649-652

DOI 10.1007/s00066-012-0151-y

Published online: 24. Juni 2012

(c) Springer-Verlag 2012
F. Wenz · Y. Abo-Madyan · G. Welzel · F.A. Giordano

University Medical Center Mannheim, University of Heidelberg, Mannheim

\title{
ABVD vs. radiotherapy in early stage Hodgkin's lymphoma
}

\section{A critical look at the NCIC HD.6 trial}

Today more than $90 \%$ of patients with early stage Hodgkin's lymphoma (HL) will be long-term survivors. Only few patients will die from the disease itself. Thus, the choice of therapy is more and more dependent on death from other causes and therapy-related (late) adverse effects. Based on their interpretation of the data from the HD.6 trial, the NCIC Clinical Trials Group and the Eastern Cooperative Oncology Group saw no difference in overall survival between radiotherapy alone and ABVD chemotherapy alone in patients with favorable risk profile. For patients with unfavorable risk profile receiving subtotal nodal radiation therapy (STNRT), they concluded that the increased number of premature deaths was related to causes other than Hodgkin's lymphoma, favoring chemotherapy over radiotherapy. However, our analysis and interpretation of the presented data raises concerns about this conclusion by the authors.

\section{Background}

Hodgkin's lymphoma (HL) is one of the most frequent malignancies of young adults although the incidence has two peaks at around 25 and 55 years [1]. Since modern therapy options have significantly improved cure rates, the vast majority of patients will survive HL long term and thus will face potential late effects of therapy, e.g., death due to secondary cancers or late cardiovascular effects.
The treatment modalities as well as the treatment intensities depend on the disease stage and, especially in early stages, on the presence of risk factors ( $\bullet$ Tab. 1). It is commonly accepted that most patients in early stages receive combined radiochemotherapy consisting of two (favorable risk) to four (unfavorable risk) cycles of ABVD or BEACOPP/ABVD and involved-field radiotherapy (RT) $[2$, 3]. Reduction of therapy-related toxicity has been not only achieved by the introduction of ABVD but also by continuously narrowing the irradiated field sizes. In this regard, subtotal nodal radiotherapy (STNRT), introduced in the early 1960s [4], has been gradually shrinking towards until today's highly circumscribed involved-field RT (or even involved-node RT) without affecting therapy efficacy $[2$, 3, 5].

Patients undergoing radiochemotherapy are at increased risk for developing secondary neoplasms, where acute myeloid leukemia (AML), myelodysplastic syndrome (MDS), and non-Hodgkin's lymphoma (NHL) occur with the highest incidence as second cancers after HL treatment $[5,6]$. Although for AML and MDS, there is a generally accepted link to the use (and dose) of DNA-destabilizing agents (e.g., alkylators or doxorubicin) [7], NHL is thought to be more related to the underlying immunosuppression rather than the drugs [8]. An increased risk of secondary solid tumors (mainly secondary breast and lung cancers) is attributable to both chemotherapy and radiotherapy [9, 10]. Where age at exposure, radiation dose and field size play an important role in defining the excess risk, a similar dose-response relationship is also recognized for secondary lung cancer with the use of alkylators $[11,12]$. However, defining the individual contributions of either chemoor radiotherapy in combined regimens is problematic [13]. Especially since most cancer patients show an increased susceptibility to develop other cancers, independent from any treatment, as can be seen for example in breast cancer patients who underwent surgery only without receiving any adjuvant therapy [14].

The recently published paper on the results of the HD.6 trial conducted by the NCIC Clinical Trials Group and the Eastern Cooperative Oncology Group reported on increased death rates from other causes than HL in patients with unfavorable risk profile undergoing (now outdated) STNRT [15].

The authors conclude that ".. the rate of long term survival is higher with ABVD alone than with treatment that includes STNRT, particularly when STNRT is combined with two cycles of ABVD therapy... the extent of radiotherapy is very likely to have contributed to the excess deaths...." We have read the paper with interest and would like to point out why we are concerned about the conclusions drawn by the authors and David Straus in his accompanying editorial [5]. 
Tab. 1 Various definitions of risk factors in early stage Hodgkin's lymphoma

\begin{tabular}{|c|c|c|c|c|c|c|}
\hline \multirow[t]{2}{*}{ Criteria } & \multicolumn{3}{|c|}{ Favorable risk } & \multicolumn{3}{|c|}{ Unfavorable risk } \\
\hline & EORTC & HD.6 & GHLSG & EORTC & HD.6 & GHLSG \\
\hline $\begin{array}{l}\text { Involved areas, } \\
n\end{array}$ & $\leq 3$ & $\leq 3$ & $<3$ & $>3$ & $>3$ & $\geq 3$ \\
\hline Age, years & $\leq 50$ & $<40$ & n.d. & $>50$ & $\geq 40$ & n.d. \\
\hline $\begin{array}{l}\text { Mediastinal } \\
\text { mass }\end{array}$ & $\begin{array}{l}\text { None or } \\
\text { small }\end{array}$ & n.d. & $\begin{array}{l}<1 / 3 \text { of thoracic } \\
\text { diameter }\end{array}$ & Large & & $\begin{array}{l}>1 / 3 \text { of thoracic } \\
\text { diameter }\end{array}$ \\
\hline Extranodal sites & n.d. & n.d. & No & n.d. & n.d. & Yes \\
\hline $\begin{array}{l}\text { ESR (no B-symp- } \\
\text { toms) }\end{array}$ & $\begin{array}{l}\leq 50 \\
\mathrm{~mm} / \mathrm{h}\end{array}$ & \multirow[t]{2}{*}{$\begin{array}{l}<50 \\
\mathrm{~mm} / \mathrm{h}\end{array}$} & $<50 \mathrm{~mm} / \mathrm{h}$ & $\begin{array}{l}>50 \\
\mathrm{~mm} / \mathrm{h}\end{array}$ & \multirow[t]{2}{*}{$\begin{array}{l}\geq 50 \\
\mathrm{~mm} / \mathrm{h}\end{array}$} & $\geq 50 \mathrm{~mm} / \mathrm{h}$ \\
\hline $\begin{array}{l}\text { ESR (B-symp- } \\
\text { toms) }\end{array}$ & $\begin{array}{l}\leq 30 \\
\mathrm{~mm} / \mathrm{h}\end{array}$ & & $\leq 30 \mathrm{~mm} / \mathrm{h}$ & $\begin{array}{l}>30 \\
\mathrm{~mm} / \mathrm{h}\end{array}$ & & $>30 \mathrm{~mm} / \mathrm{h}$ \\
\hline
\end{tabular}

GHLSG German Hodgkin Lymphoma Study Group, n.d. not defined, ERS erythrocyte sedimentation rate.

Tab. 2 Second cancers observed in the HD.6 trial

\begin{tabular}{|lllll}
\hline Risk profile & Therapy & $\begin{array}{l}\text { Total } \\
\mathbf{n}\end{array}$ & $\begin{array}{l}\text { SC } \\
\mathbf{n}(\%)\end{array}$ & $\begin{array}{l}\text { Deaths by SC } \\
\mathbf{n}(\%)\end{array}$ \\
\hline Favorable & ABVD only & 59 & n.p. & 0 \\
\cline { 2 - 5 } & STNRT $^{a}$ & 64 & $4(6 \%)$ & $1(2 \%)$ \\
\hline Unfavorable & ABVD only $^{\text {S }}$ & 137 & n.p. & $4(3 \%)$ \\
\cline { 2 - 5 } & STNRT $^{a}$ & 139 & $19(14 \%)$ & $9(6 \%)$ \\
\hline & ABVD only $^{\text {STNRT }}$ & 196 & $10(5 \%)$ & $4(2 \%)$ \\
\cline { 2 - 5 } & STNR $^{a}$ & 203 & $23(11 \%)$ & $10(5 \%)$ \\
\hline
\end{tabular}

n.p. not published, SC second cancers

a $14 \%$ of patients also received chemotherapy.

\section{Rationale and design of the HD.6 trial}

The rationale of the Hodgkin's Disease. 6 (HD.6) trial (NCT00002561) was to determine whether HL patients treated with ABVD alone show equal long-term survival rates as those treated with STNRT or combined ABVD-STNRT. It was started in 1994 and closed in 2002, whenbased on results from the German Hodgkin Lymphoma Study Group (GHLSG)involved-field RT was accepted to be a superior treatment approach to STNRT.

Furthermore, according to the decision of the trial committee and the data and safety monitoring board, the date of the final statistical analyses was changed from December 2015 to December 2010 because 31 deaths had occurred in March 2010, and the trial committee did not expect that the trial would reach its end point of 56 deaths by 2015 .

Patients with histologically confirmed, previously untreated, Ann Arbor-stage IA or IIA non-bulky (mass $<10 \mathrm{~cm}$ ) HL were included. Before randomization, patients were stratified into favorable and unfavor- able risk cohorts (• Tab. 1). Risk-stratified patients were then randomly assigned to one of two treatment arms, whereas one arm contained a treatment including RT (favorable profile: STNRT alone, unfavorable profile: 2 cycles of ABVD + STNRT) and the other arm included ABVD (46 cycles) as single treatment modality (• Fig. 1a). Patients assigned to ABVD alone who were not achieving complete remission (CR) or unconfirmed complete remission ( $\mathrm{uCR}$ ) after two cycles received four more cycles of ABVD. In case of disease progression during treatment with ABVD alone, radiotherapy was optionally applicable. Primary end point of the study was the 12-year overall survival (OS). Secondary end points were freedom from disease progression (FFDP), second cancer (SC) rate and cardiac events.

\section{HD.6 Radiotherapy details}

Initially after randomization of patients into the RT group (favorable risk cohort) or 4-6 weeks after two cycles of chemotherapy (unfavorable risk cohort), the patients received $35 \mathrm{~Gy}$ in 20 fractions of
1.75 Gy to each the supradiaphragmatic lymph node areas ("mantle field"), the spleen and the upper abdominal lymph nodes ( $\bullet$ Fig. 2b). Of importance, the RT fields in the favorable group were larger (lower border was L4) than in the unfavorable risk cohort (L2).

\section{Overall and progression-free survival}

The authors reported equal OS and FFDP rates of patients with favorable risk (FR) profile when comparing the ABVD only (OS 98\%; FFDP 89\%) to the STNRT only (OS 98\%; FFDP 87\%) group. In contrast, patients with unfavorable risk (UFR) profile receiving ABVD only had a better OS (92\%) than those receiving additional STNRT (81\%) although FFDP was about $8 \%$ superior after STNRT-ABVD. In total, 11 deaths occurred among UFR patients in the ABVD alone group and 23 in the combined group. Looking closer at the causes, one notes 5 "other" causes of death (including 1 Alzheimer's, 1 suicide, 1 drowning, 1 respiratory failure, and 1 unknown) and 3 deaths due to infection during therapy in the STNRT-ABVD group that were considered for the analysis (in conformity with an intention-to-treat analysis).

Yet, since at least 4 (respiratory failure may be radiation-related) of the 5 "other" causes of death are clearly not therapy related (and by far not radiation-related), an additional analysis excluding these deaths would have been more logical and would have resulted in 11 vs. 19 deaths in the unfavorable group and, in total (FR and UFR combined), 12 vs. 20 "other" deaths $\left(\chi^{2}\right.$ test, $\left.\mathrm{p}=0.17\right)$. Of note, the resulting insignificant difference in the "corrected" OS is accompanied by a highly significant higher FFDP rate at 12 years when RT is added (94\% with RT vs. $86 \%$ without RT; $\mathrm{p}=0.006)$.

\section{Second cancers}

On first sight and due to the way that the data are presented, the rates of second cancers (SC) appear higher when STNRT is given alone or combined with ABVD as compared to ABVD alone (• Tab. 2). A detailed look demonstrates that this is caused only by an increased incidence of 


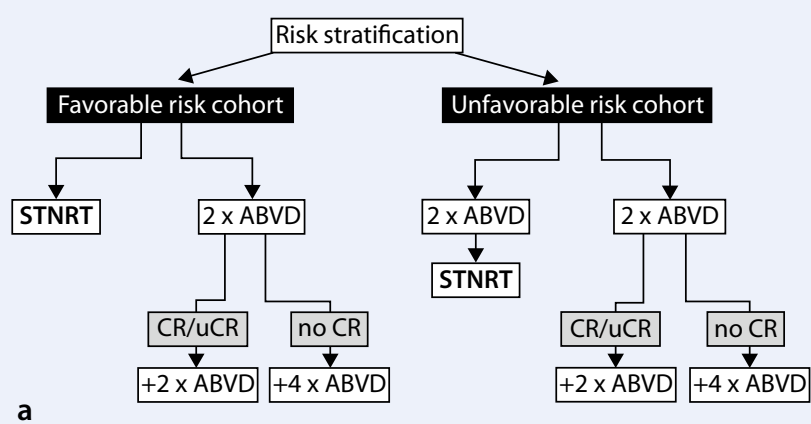

a

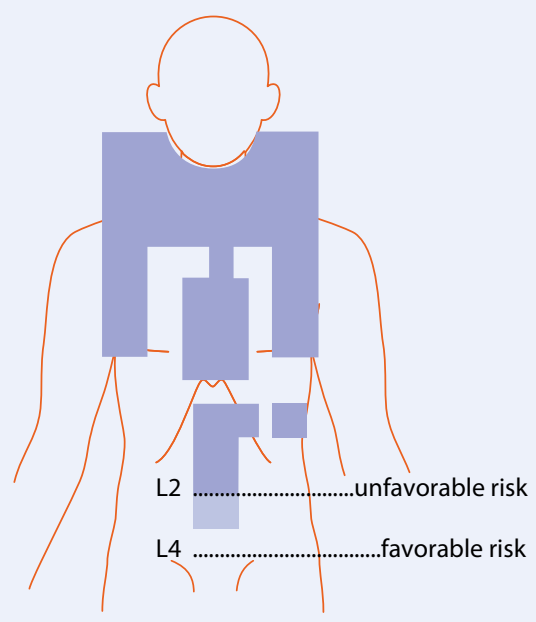

Fig. $1<$ a Study design: following risk stratification, patients were randomly assigned to a regimen containing ABVD chemotherapy alone or radiotherapy (alone or combined). b Radiotherapy fields applied in the two risk cohorts: target volumes were the supradiaphragmatic lymph node areas, the spleen and the upper abdominal lymph nodes. The RT fields in the favorable group were larger (lower border at L4) than in the unfavorable risk cohort (L2)

SC in the unfavorable cohort receiving STNRT + ABVD.

The addition of two cycles of ABVD to $\mathrm{RT}$ in the unfavorable group resulted in an increase of SC when compared to the favorable group receiving STNRT alone (19 vs. 4 ) and deaths by SC (9 vs. 1). Of note, the unfavorable cohort received RT to smaller fields (lower field edge at L2, compared to L4 in the favorable cohort) with more patients receiving "less than protocol radiotherapy" ( $9 \%$ vs. $3 \%$ ) than in the favorable cohort, and thus, based on the well-known age-, doseand volume-effect relationship for second cancer induction after RT [16], the reasons for these counterintuitive findings (i.e. higher SC risk in older patients with smaller RT volumes and lower RT intensity) remain open to speculation. In addition, observation studies on atomic bomb survivors showed colon, lung and stomach to be prime sites for SC after RT [17, 18]. None of the $4 \mathrm{SC}$ in the radiation-only cohort with favorable risk (2 lymphoma, cervical cancer and melanoma) was typical for the used RT fields (although a de- tailed location of the lymphomas and the melanoma were not given). Recent studies on patients with various cancers that received high-dose radiation therapy revealed a $2.2 \%$ (dose-dependent) probability of developing a second cancer in the irradiated field [19] and analyses of the SEER registry revealed that an estimated $8 \%$ of SC developing in patients are attributable to radiation treatment for the original cancer [20].

The unfavorable cohort treated with ABVD alone in the NCIC HD.6 trial showed higher rates of deaths by SC than the corresponding favorable cohort (4 vs. 0) receiving ABVD alone in contrast to other studies (with shorter follow ups) $[2,21]$. Unfortunately, the detailed numbers on how the 10 SC cases split in both groups were not given in either the paper or the supplement. Since chemotherapy was applied with the same intensity $(90 \%$ of all ABVD-only patients, of both the favorable and unfavorable cohort, achieved $\mathrm{CR} / \mathrm{uCR}$ and received only $4 \mathrm{xABVD}$ ), this higher rate clearly indicates that second cancer death is more likely associat- ed with the presence of risk-factors in the UFR group and not with therapy itself.

\section{Comments and conclusion}

The HD.6 study is doubtless a well-conducted and very important study of highest interest. Though, in our opinion, the data workup and presentation in this paper and the timing of the (early) final statistical analysis (5 new deaths were observed from March 2010 to December 2010) can be questioned and the conclusions drawn by the authors as well as by David Straus [5] can be challenged.

One may as well conclude that it is unlikely that radiotherapy is the predominant cause for the observed events in the unfavorable-risk group. It rather appears that age (risk factor $>39$ years), paraneoplastic effects (a risk factor was increased ESR, indicating some systemic effects) or the interaction of ABVD with radiotherapy may play more important roles. The unexplained high risk for SC death after ABVD and ABVD/RT in HL patients who are older than 40, have a high ESR, or multiple involved locations should have been highlighted in the paper.

Until the underlying causes are understood, patients with unfavorable risk profile should be carefully advised about these effects if large radiation treatment fields and combined modality treatments are needed, which in the era of improved imaging-based staging may not be necessary. Since the treatment modalities have significantly changed over the years and the outdated STNRT is replaced by highly precise involved field or involved noderadiotherapy, this study has to be read and interpreted with caution. Moreover, there should be awareness that the higher cure rates justify the use of RT since most second cancers are developing much later than the (prevented) local recurrences [10].

In summary, we suggest a potential alternative conclusion:

Compared to ABVD alone, subtotal nodal radiotherapy alone (STNRT) showed equivalent rates of overall survival, progression-free survival, second cancers and cardiac events in patients with early stage HL and favorable risk profile. The addition of ABVD to STNRT in- 
creased the risk of SC (and CE) considerably as compared to STNRT alone despite smaller RT volumes (lower borders: L2 vs. L4) and more patients receiving less than protocol radiation ( $9 \%$ vs. $3 \%$ ) in the unfavorable risk group (risk factors: $>39$ years, $\mathrm{ESR} \geq 50 \mathrm{~mm} / \mathrm{h}$, histology $>3$ sites). Based on the well-known age-, dose- and volume-effect relationship for SC induction after RT, the reasons for these counterintuitive findings remain open to speculation but may be more likely disease- than therapy-associated.

\section{Corresponding address}

\section{Prof. Dr. F. Wenz}

University Medical Center Mannheim, University of Heidelberg

Theodor-Kutzer-Ufer 1-3, 68167 Mannheim

Germany

frederik.wenz@umm.de

\section{References}

1. www.seer.cancer.gov; based on the November 2008 submission

2. Engert A, Plütschow A, Eich HT et al (2010) Reduced treatment intensity in patients with early-stage Hodgkin's lymphoma. N Engl J Med 363:640-652

3. von Tresckow B, Plütschow A, Fuchs M et al (2012) Dose-intensification in early unfavourable Hodgkin's lymphoma: final analysis of the German Hodgkin Study Group HD14 Trial. J Clin Oncol 30:907-913

4. Kaplan H (1962) The radical radiotherapy of regionally localized Hodgkin's disease. Radiology 78 : 553-561

5. Straus DJ (2012) Chemotherapy alone for early-stage Hodgkin's lymphoma. N Engl J Med 366:470-471

6. Vardiman JW, Thiele J, Arder DA et al (2009) The 2008 revision of the World Health Organization (WHO) classification of myeloid neoplasms and acute leukemia: rationale and important changes. Blood 114:937-951

7. Leone G, Pagano L, Ben-Yehuda D et al (2007) Therapy-related leukemia and myelodysphasia: susceptibility and incidence. Haematologica 92:1389-1398

8. de Jong D (2005) Molecular pathogenesis of follicular lymphoma: a cross talk of genetic and immunologic factors. J Clin Oncol 23:6358-6363

9. Slanina J, Heinemann F, Henne K et al (1999) Second malignancies after therapy of Hodgkin's disease: the Freiburg collective 1940 to 1991. Strahlenther Onkol 175:154-161

10. Dörr W, Herrmann T (2008) Second tumors after oncologic treatment. Strahlenther Onkol 184:6772

11. Swerdlow AJ, Barber JA, Hudson GV et al (2000) Risk of second malignancy after Hodgkin's disease in a collaborative British cohort: the relation to age at treatment. J Clin Oncol 18:498-509
12. Travis LG, Gospodarowicz MK, Curtis RE et al (2002) Lung cancer following chemotherapy and radiotherapy for Hodgkin's disease. J Natl Cancer Inst 94:182-192

13. Schulz U, Gokel JM, Poleska W (2000) Soft tissue sarcomas after radiation treatment for breast cancer. Three case studies and review of literatre. Strahlenther Onkol 176:144-149

14. Martin MG, Welch JS, Luo J et al (2009) Therapy related acute myeloid leukemia in breast cancer survivors, a population-based study. Breast Cancer Res Treat 118:593-598

15. Meyer RM, Gospodarowicz MK, Connors JM et al (2012) ABVD alone versus radiation-based therapy in limited-stage Hodgkin's lymphoma. N Engl J Med 366:399-408

16. Kaatsch P, Debling D, Blettner M, Spix C (2009) Second malignant neoplasms after childhood cancer in Germany - results from the long-term follow-up of the German Childhood Cancer Registry. Strahlenther Onkol 185 (Suppl 2):8-10

17. Preston DL, Ron E, Tokuoka S et al (2007) Solid cancer incidence in atomic bomb survivors: 19581998. Radiat Res 168:1-64

18. Hall EJ (2006) Intensity-modulated radiation therapy, protons, and the risk of second cancers. Int J Radiat Oncol Biol Phys 65:1-7

19. Welte B, Suhr P, Bottke D et al (2010) Strahlenther Onkol 186:174-179

20. Berrington de Gonzalez A, Curtis RE, Kry SF et al (2011) Proportion of second cancers attributable to radiotherapy treatment in adults: a cohort study in the US SEER cancer registries. Lancet Oncol 12:353-360

21. Fermé C, Eghbali H, Meerwaldt JH et al (2007) Chemotherapy plus involved-field radiation in earlystage Hodgkin's disease. N Engl J Med 357:19161927 